\title{
Reduction in cerebral blood flow in areas appearing as white matter hyperintensities on magnetic resonance imaging
}

\author{
Adam M. Brickman,*, Amir Zahraa, Jordan Muraskin', Jason Steffener ${ }^{\mathrm{a}}$, Christopher M. Holland ${ }^{\mathrm{b}, \mathrm{c}}$, \\ Christian Habeck ${ }^{\mathrm{a}}$, Ajna Borogovac ${ }^{\mathrm{d}}$, Marco A. Ramos, ${ }^{\mathrm{a}}$, Truman R. Brown ${ }^{\mathrm{d}, \mathrm{e}}$, Iris Asllanie, Yaakov Stern ${ }^{\mathrm{a}}$ \\ ${ }^{a}$ Taub Institute for Research on Alzheimer's Disease and the Aging Brain, College of Physicians and Surgeons, Columbia University, New York, NY, United States \\ ${ }^{b}$ Center for Neurological Imaging, Brigham and Women's Hospital, Harvard Medical School, Boston, MA, United States \\ 'Department of Anatomy and Neurobiology, Boston University School of Medicine, Boston, MA, United States \\ ${ }^{\mathrm{d}}$ Department of Biomedical Engineering, Columbia University, New York, NY, United States \\ e Department of Radiology, College of Physicians and Surgeons, Columbia University, New York, NY, United States
}

\section{A R T I C L E I N F O}

\section{Article history:}

Received 1 July 2008

Received in revised form 10 October 2008

Accepted 13 November 2008

\section{Keywords:}

White matter hyperintensities

Arterial spin labeling

MRI

\begin{abstract}
A B S T R A C T
The purpose of this study was to examine cerebral blood flow (CBF) as measured by arterial spin labeling (ASL) in tissue classified as white matter hyperintensities (WMH), normal appearing white matter, and grey matter. Seventeen healthy older adults received structural and ASL MRI. Cerebral blood flow was derived for three tissue types: WMH, normal appearing white matter, and grey matter. Cerebral blood flow was lower in $\mathrm{WMH}$ areas relative to normal appearing white matter, which in turn, was lower than grey matter. Regions with consistently lower CBF across individuals were more likely to appear as WMH. Results are consistent with an emerging literature linking diminished regional perfusion with the risk of developing WMH.
\end{abstract}

(C) 2008 Elsevier Ireland Ltd. All rights reserved.

\section{Introduction}

White matter hyperintensities (WMH) are areas of increased signal intensity detected on T2-weighted magnetic resonance imaging (MRI) scans. These lesions are common among older adults (Brickman et al., 2008a) and are thought to reflect small vessel vascular disease or $\beta$-amyloid peptide deposition (Gurol et al., 2006). Increased $\mathrm{WMH}$ volume is associated with degree of cognitive impairment among neurologically healthy older adults (GunningDixon and Raz, 2000) and is predictive of rate of cognitive decline among those with Alzheimer's disease (Brickman et al., 2008b). Although these associative findings have been consistently reported, the functional significance of $\mathrm{WMH}$ is unclear.

Increases in total or frontal WMH volume have been associated with decreases in relative glucose metabolism in frontal regions among healthy older adults (DeCarli et al., 1995). In a longitudinal analysis, individuals with increasing severity of WMH over an 8-year period had concomitant increases in relative cerebral blood flow (CBF) in temporal areas and anterior cingulate and decreases in relative CBF in more posterior areas (Kraut et al., 2008). Further, increased severity of WMH was not associated with total parenchymal CBF in a cross-sectional

\footnotetext{
* Corresponding author. Taub Institute for Research on Alzheimer's, Disease and the Aging Brain, Box 16, College of Physicians and Surgeons, Columbia University, 630 West 168th Street, New York, NY 10032, United States. Tel.: +1 212342 1348; fax: +1 212342 1838.

E-mail address: amb2139@columbia.edu (A.M. Brickman).
}

analysis, but decreases in CBF over time increased the risk of developing periventricular WMH (ten Dam et al., 2007). Holland and colleagues recently showed that WMH frequency among healthy older adults and those with $\mathrm{AD}$ or cerebral amyloid angiopathy is greater in regions with relatively lower perfusion among younger adults (Holland et al., 2008). These findings raise the possibility that lower regional perfusion increases the risk of developing WMH.

In the current study, we sought to extend these findings by examining arterial spin labeling (ASL)-derived CBF in areas appearing as $\mathrm{WMH}$ on $\mathrm{T} 2$-weighted fluid attenuated inverse recovery (FLAIR) MRI scans in comparison to CBF in normal appearing white matter (NAWM) and grey matter among neurologically healthy older adults. Two previous studies used perfusion-weighted MRI and found a decline in blood flow in areas appearing as WMH on T2weighted images compared with NAWM (Marstrand et al., 2002; Sachdev et al., 2004). Thus, we hypothesized that CBF would be decreased in areas appearing as WMH compared with CBF in NAWM and grey matter.

\section{Methods \\ 2.1. Subjects}

Seventeen older adults ranging in age from 61 to 70 (mean + S.D. $=64.94 \pm 2.95$ ) comprised the study sample. There were 9 women (53\%) and the average number of years of formal education was 16.29 (S.D. $=1.72$ ). Men and women did not differ in age $(t(15)=0.235$, $P=0.817)$ or number of years of education $(t(15)=0.371, P=0.716)$. All participants were right-handed and spoke English. By self-report and interview, participants did not have past or current histories of medical, neurological, psychiatric disorders or treatment with 
psychoactive medication. Participants were screened for dementia with interview and psychometric assessment (Mattis, 1988); none met diagnostic criteria. Subjects were recruited for participation in an ongoing functional magnetic resonance imaging (fMRI) study of working memory and aging. The study was approved by a local ethics committee, and all participants gave written informed consent to participate in the study.

\subsection{Magnetic resonance imaging}

\subsubsection{Image acquisition}

All neuroimaging took place in a single session on a $1.5 \mathrm{~T}$ Philips Intera scanner. Standard high-resolution T1-weighted anatomical images were acquired in the axial plane using the following parameters: $F O V=230 \times 230 \mathrm{~mm}$, matrix $=256 \times 256$, slice thickness $=1.5 \mathrm{~mm}$, TR/TE $=25 \mathrm{~ms} / 3 \mathrm{~ms}$. T2-weighted FLAIR images were acquired for $\mathrm{WMH}$ quantification with the following parameters: FOV $=250 \times 250 \mathrm{~mm}$ matrix $=256 \times 192$, slice thickness $=3 \mathrm{~mm}, \mathrm{TR} / \mathrm{TE}=11,000 \mathrm{~ms} / 144 \mathrm{~ms}$. Detailed description of acquisition of continuous arterial spin labeling (CASL) perfusion images is provided elsewhere (Asllani et al., 2008). Briefly, 30 spin echo, echo planar (EPI) CASL control and labeled images were acquired axially (inferior to superior) with a labeling duration of $2000 \mathrm{~ms}$, post-labeling delay of $800 \mathrm{~ms}$, in-plane resolution $3.4 \times 3.8 \mathrm{~mm}$, slice thickness of $7.5 \mathrm{~mm}$, FOV $=220 \times 220 \mathrm{~mm}$, and TR/TE $=5000 \mathrm{~ms} / 35 \mathrm{~ms}$. Adiabatic inversion labeling of the water spins and correction for off-resonance saturation were implemented following procedures put forth by Alsop and Detre (Alsop and Detre, 1996; see Asllani et al., 2008).

\subsubsection{MRI data preprocessing}

Preprocessing steps were implemented with the SPM99 software package (Wellcome Department of Cognitive Neurology) with in-house developed software written in MatLab (Mathworks, Natick, MA, USA), described fully in a previous report (Asllani et al., 2007). All CASL and T1-weighted images were coregistered to the first CASL image in the acquisition series. The T1-images were spatially normalized using $7 \times 8 \times 7$ nonlinear basis functions into Talairach space defined by the Montreal Neurologic Institute (MNI) and segmented into grey matter, white matter, and cerebral spinal fluid (CSF) posterior probability images. These tissue segment probability maps were thresholded at $0.80(80 \%)$ to include only voxels with high probability of belonging to a tissue class and reduce the potential influence of partial volume effects. The spatial transformation was applied to the coregistered CASL and tissue segment images; these procedures ensured that all CASL and anatomical images were aligned in the same stereotactic space.

\subsubsection{Computation of cerebral blood flow}

For computation of cerebral blood flow (CBF), the paired control/label images in Talairach space were used to calculate percent change images following a modified two-compartment formula, as detailed elsewhere (Asllani et al., 2008). In each voxel, $\mathrm{CBF}$ values were weighted by the tissue type (i.e., grey matter, white matter, CSF) posterior probability as determined from the T1-weighted image tissue segment. Finally, CBF images were smoothed with a $6-\mathrm{mm}$ spatial kernel.

\subsubsection{Quantification of white matter hyperintensities}

For quantification of WMH, FLAIR images were imported into the publically available software package MRICro (Rorden and Brett, 2000), and WMH volumes were determined following a three-step process put forth by Gurol et al. (2006). First, based on a priori knowledge of the distribution of voxel intensities and visual inspection of each individual image, we determined the intensity threshold that labeled voxels appearing as hyperintense. Second, on a slice-by-slice basis, regions of interest (ROIs) were manually traced to define gross areas containing WMH while excluding nonWMH areas that were labeled in step 1 (e.g., dermal fat). Third, the intersection of the labeled voxels in Step 1 and the manual ROIs yielded the volume of WMH in $\mathrm{cm}^{3}$. Further, binary image maps were created such that all voxels labeled as WMH were assigned the value 1 and all remaining voxels were assigned the value 0 . These maps were spatially normalized into Talairach space through application of the transformation algorithm derived from the normalization of the T1-weighted image.

\subsection{Outcome measures}

Three primary measures were determined for statistical analysis for each subject. First, the segmented grey matter image was coregistered to the ASL image, and the mean CBF was calculated from all voxels defined as greater than $80 \%$ grey matter. Second, the binary WMH map was coregistered to the ASL image, and WMH CBF was defined as the mean over all voxels labeled as WMH. Third, the segmented white matter image was coregistered to the ASL image, and the mean CBF was calculated from all voxels defined as greater than $80 \%$ white matter after removing those voxels identified as WMH (i.e., NAWM).

\section{Results}

Participants in the current study had a relatively mild degree of WMH burden (mean \pm S.D. WMH volume $=4.631 \pm 4.836 \mathrm{~cm}^{3}$, median $=2.00$, range $=0.10$ to 16.90 ). A general linear model was constructed to evaluate differences in $\mathrm{CBF}$ among voxels classified as

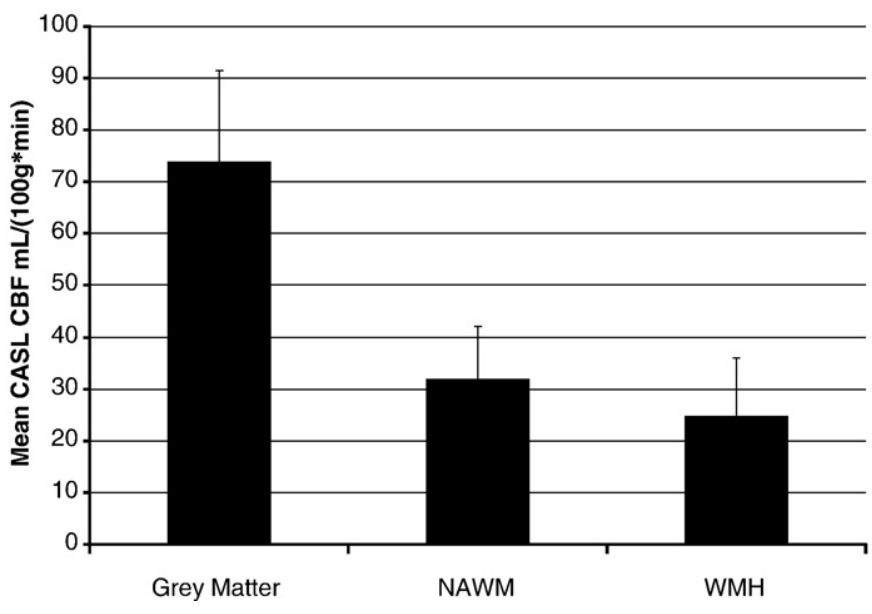

Fig. 1. Mean differences in CASL-determined blood flow in grey matter regions, in normal appearing white matter (NAWM), and in areas with white matter hyperintensities (WMH). Error bars are standard deviations. All pairwise comparisons are statistically significant $(P<0.001)$.

WMH, NAWM, and grey matter. Cerebral blood flow in each of these regions was treated as a repeated measure (i.e., within-subjects factor). There was a significant overall difference in $\mathrm{CBF}$ among these regions $(F(2,32)=185.61, P<0.001)$. Pairwise comparisons indicated that CBF was significantly lower in WMH than in NAWM, which, in turn, was significantly lower than CBF in grey matter $(P \leq 0.001$ for all pairwise comparisons). These results are displayed in Fig. 1. Further, the non-parametric Wilcoxon signed ranks test confirmed significant differences among groups (all group comparisons, $P<0.004$ ). Fig. 2 displays an image in which voxels labeled as $\mathrm{WMH}$ in at least five subjects are superimposed on the mean CBF image derived by averaging the CASL images from all subjects.

We examined whether the mean CBF value varied as a function of the likelihood that any given voxel was labeled as WMH. To do this analysis, we created a conjunction map of all individual WMH masks from individual subjects in standardized anatomical space. We grouped voxels by frequency and compared mean CBF values across frequency values, which ranged from 1 to 15 (note that no single voxel was labeled as a WMH in all 17 subjects). As displayed in Fig. 3, there was a subtle, but significant $(F(14,21787)=174.439, P<0.001)$ linear decline ( $P$ for linear trend $=0.045$ ) across frequency groups. Next we calculated the standard deviation and coefficient of variation (a normalized metric of dispersion, $\sigma / \mu$ ) for mean CBF values in each frequency group and correlated those values against frequency. Decreases in both the standard deviation (Spearman's rho $=-0.889$, $P<0.001$ ) and the coefficient of variation (Spearman's rho $=-0.796$, $P<0.001$ ) were strongly related to increased frequency. Taken together, these findings suggest that regions with consistently low CBF across individuals are more likely to appear as WMH.

\section{Discussion}

In the current study, we compared CASL-derived mean CBF across three tissue types - areas appearing as WMH on T2-weighted FLAIR MRI, NAWM, and grey matter - in well-screened, neurologically healthy older adults. Findings indicated that CBF was significantly reduced in areas appearing as WMH compared with both NAWM and grey matter. These results are consistent with previous reports in demonstrating an association between $\mathrm{WMH}$ and reduced blood flow, and replicate findings suggesting that this association is specific to the tissue appearing as WMH itself (Marstrand et al., 2002; Sachdev et al., 2004).

White matter hyperintensities are common findings on T2weighted images among older adults. Although WMH were previously 
thought to lack clinical significance, several studies now confirm that they are associated with poorer cognitive function, may put individuals at risk for the development of dementia, and are more prevalent among certain psychiatric diagnoses. Results from the current study suggest that these associations may be due partially to a reduction in perfusion in areas appearing as WMH. However, causal directionality is difficult to infer from cross-sectional studies. It is possible, for example, that small vascular lesions appear first as WMH and then have a secondary effect on underlying perfusion or that the two phenomena occur concomitantly without a causal link. A recent study by Holland et al. (2008) speaks against this possibility. They compared the spatial distribution of WMH among healthy older adults and those with $\mathrm{AD}$ or cerebral amyloid angiopathy as it related to a normative atlas of perfusion values derived from neurologically healthy, younger adults and found that WMH frequency was greater in regions with lower normative perfusion values. Similarly, O'Sullivan et al. (2002) showed that CBF was reduced in periventricular NAWM among patients with ischemic leukoaraiosis compared with controls, suggesting that diminished regional white matter perfusion may be a "risk factor" for the development of WMH. Further, a recent report showed an inverse association between cardiac output and subcortical WMH volume, suggesting that systemic hypoperfusion may increase the risk for development of WMH (Jefferson et al., 2007). Future longitudinal or serial MRI studies are required to determine the exact causal relationship between diminished perfusion and $\mathrm{WMH}$.

Visual inspection of Fig. 2 shows reduced CBF in areas labeled as $\mathrm{WMH}$ in at least five participants from the current study. The figure also reveals that areas that are most vulnerable to the development of $\mathrm{WMH}$, namely periventricular regions that are confluent with the lateral ventricles, appear to have a more profound reduction in blood flow than white matter that is distributed in deeper cortical areas. Findings displayed in Fig. 3 suggest that areas that are of consistently low CBF are most likely to be labeled as WMH across individuals.

Two potential limitations should be pointed out. First, atrophyassociated ventricular enlargement may have increased the likelihood of contamination by the CSF of the WMH voxels. To limit this possibility, we restricted our analyses to areas with high probability of belonging to a tissue class. Second, although not visualized on T1weighted images, WMH regions may create subtle signal alterations, which might have affected tissue segmentation used for conversion to CBF of ASL percent change data. Third, it is also conceivable that tissue transit times are longer in $\mathrm{WMH}$, possibly due to associated vasculature changes. This transit time confound would cause an

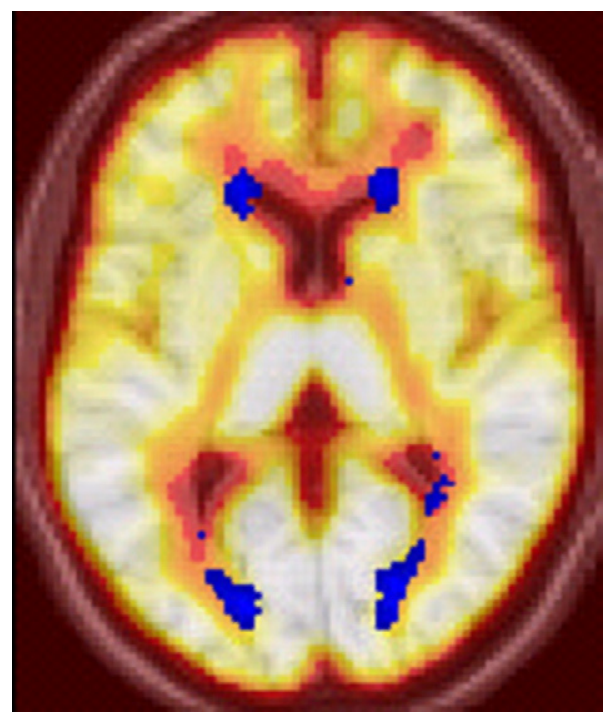

Fig. 2. Voxels labeled as WMH in at least 5 subjects (in blue) superimposed on the mean CBF image derived by the average of all subjects' CASL images.

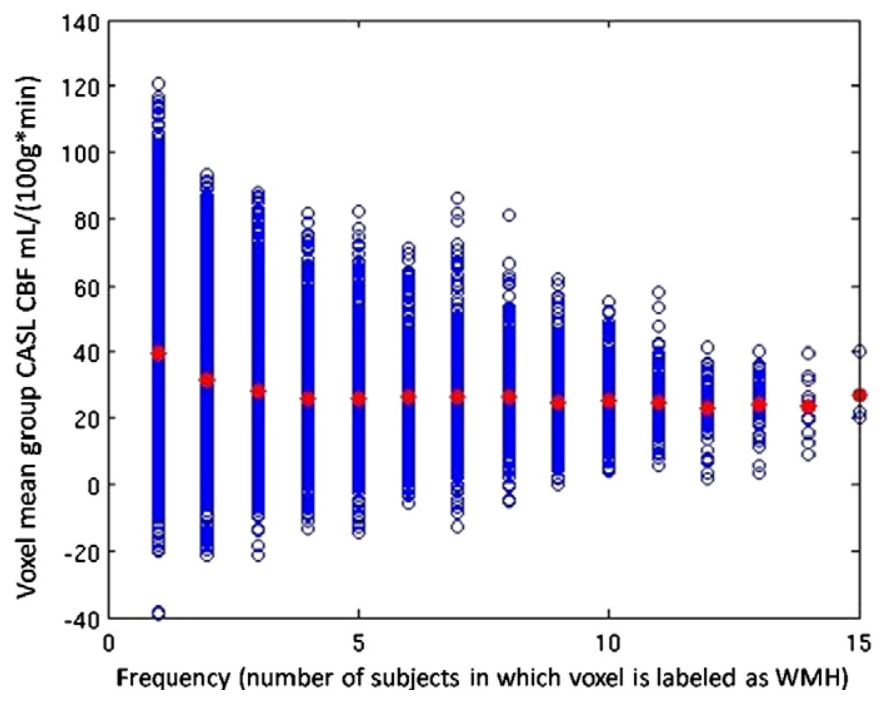

Fig. 3. Mean group $\mathrm{CBF}$ displayed as a function of the frequency (i.e., number of subjects) in which voxels are labeled as WMH. Each circle represents a single voxel. For example, a single circle in frequency $=5$ represents the total group mean CBF for a voxel that is labeled as WMH in five subjects. Note subtle decline in group mean CBF across frequency (red dot) and marked decrease in variability, suggesting that areas with consistently lower CBF are most likely to be WMH across individuals. The number of voxels included for each frequency, 1 through 15, respectively, are 12109, 3811, 1796, $1125,795,608,482,377,269,212,114,58,32,10$, and 4 . (For interpretation of the references to color in this figure legend, the reader is referred to the web version of this article.)

underestimation of CBF. Future work is needed to separate the effect of transit time from absolute $\mathrm{CBF}$ reduction in $\mathrm{WMH}$ as this would aid in our understanding of the underlying mechanism of the observed CBF reduction in $\mathrm{WMH}$.

Magnetic resonance imaging has emerged as having the utility for identification of the structural and functional correlates of cognitive aging. Converging evidence from animal and human research literature points to age-associated changes in white matter as a particularly salient feature of the aging process. These observations are consistent with a "disconnection" hypothesis of cognitive aging, which posits that integration of coordinated neural systems declines with advancing age (Andrews-Hanna et al., 2007). The current study highlights the possibility that WMH and/or their associated diminution in blood flow may contribute to this disconnection by disrupting neural communication across white matter fibers. Future studies should integrate these MRI modalities with comprehensive cognitive data and evaluate other aspects of white matter integrity.

\section{Acknowledgments}

This work was supported by National Institutes of Health grants AG029949 (AMB), AG26158 (YS), and AG026114 (CH) and by a grant 05-14586 from the Alzheimer's Association (AMB).

\section{References}

Alsop, D.C., Detre, J.A., 1996. Reduced transit-time sensitivity in noninvasive magnetic resonance imaging of human cerebral blood flow. Journal of Cerebral Blood Flow and Metabolism 16, 1236-1249.

Andrews-Hanna, J.R., Snyder, A.Z., Vincent, J.L., Lustig, C., Head, D., Raichle, M.E., Buckner, R.L., 2007. Disruption of large-scale brain systems in advanced aging. Neuron 56, 924-935.

Asllani, I., Habeck, C., Scarmeas, N., Borogovac, A., Brown, T.R., Stern, Y., 2008. Multivariate and univariate analysis of continuous arterial spin labeling perfusion MRI in Alzheimer's disease. Journal of Cerebral Blood Flow and Metabolism 28 (4), 725-736.

Brickman, A.M., Honig, L.S., Scarmeas, N., Tatarina, O., Sanders, L., Albert, M.S., Brandt, J., Blacker, D., Stern, Y., 2008b. Measuring cerebral atrophy and white matter hyperintensity burden to predict the rate of cognitive decline in Alzheimer disease. Archives of Neurology 65, 1202-1208.

Brickman, A.M., Schupf, N., Manly, J.J., Luchsinger, J.A., Andrews, H., Tang, M.X., Reitz, C., Small, S.A., Mayeux, R., DeCarli, C., Brown, T.R., 2008a. Brain morphology in older 
African Americans, Caribbean Hispanics, and whites from northern Manhattan. Archives of Neurology 65, 1053-1061.

DeCarli, C., Murphy, D.G., Tranh, M., Grady, C.L., Haxby, J.V., Gillette, J.A., Salerno, J.A., Gonzales-Aviles, A., Horwitz, B., Rapoport, S.I., Schapiro, M.B., 1995. The effect of white matter hyperintensity volume on brain structure, cognitive performance, and cerebral metabolism of glucose in 51 healthy adults. Neurology 45, 2077-2084.

Gunning-Dixon, F.M., Raz, N., 2000. The cognitive correlates of white matter abnormalities in normal aging: a quantitative review. Neuropsychology 14 , 224-232.

Gurol, M.E., Irizarry, M.C., Smith, E.E., Raju, S., Diaz-Arrastia, R., Bottiglieri, T., Rosand, J., Growdon, J.H., Greenberg, S.M., 2006. Plasma beta-amyloid and white matter lesions in AD, MCI, and cerebral amyloid angiopathy. Neurology 66, 23-29.

Holland, C.M., Smith, E.E., Csapo, I., Gurol, M.E., Brylka, D.A., Killiany, R.J., Blacker, D., Albert, M.S., Guttmann, C.R., Greenberg, S.M., 2008. Spatial distribution of whitematter hyperintensities in Alzheimer disease, cerebral amyloid angiopathy, and healthy aging. Stroke 39 (4), 1127-1133.

Jefferson, A.L., Tate, D.F., Poppas, A., Brickman, A.M., Paul, R.H., Gunstad, J., Cohen, R.A., 2007. Lower cardiac output is associated with greater white matter hyperintensities in older adults with cardiovascular disease. Journal of the American Geriatrics Society 55, 1044-1048.

Kraut, M.A., Beason-Held, L.L., Elkins, W.D., Resnick, S.M., 2008. The impact of magnetic resonance imaging-detected white matter hyperintensities on longitudinal changes in regional cerebral blood flow. Journal of Cerebral Blood Flow and Metabolism 28, 190-197.

Marstrand, J.R., Garde, E., Rostrup, E., Ring, P., Rosenbaum, S., Mortensen, E.L., Larsson, H.B., 2002. Cerebral perfusion and cerebrovascular reactivity are reduced in white matter hyperintensities. Stroke 33, 972-976.

Mattis, S., 1988. Dementia Rating Scale (DRS). Psychological Assessment Resources, Odessa, FL.

O'Sullivan, M., Lythgoe, D.J., Pereira, A.C., Summers, P.E., Jarosz, J.M., Williams, S.C., Markus, H.S., 2002. Patterns of cerebral blood flow reduction in patients with ischemic leukoaraiosis. Neurology 59, 321-326.

Rorden, C., Brett, M., 2000. Stereotaxic display of brain lesions. Neurology 12, 191-200.

Sachdev, P., Wen, W., Shnier, R., Brodaty, H., 2004. Cerebral blood volume in T2weighted white matter hyperintensities using exogenous contrast based perfusion MRI. Journal of Neuropsychiatry and Clinical Neurosciences 16, 83-92.

ten Dam, V.H., van den Heuvel, D.M., de Craen, A.J., Bollen, E.L., Murray, H.M. Westendorp, R.G., Blauw, G.J., van Buchem, M.A., 2007. Decline in total cerebral blood flow is linked with increase in periventricular but not deep white matter hyperintensities. Radiology 243, 198-203. 